\title{
Dynamics of Circulating Tumor Cells Early After Targeting Therapy to Human EGFR-mutated Lung Cancers and HER2 Gene-amplified Gastric Cancers in Mice
}

\author{
AKIHIRO ITO ${ }^{1,2,3}$, HAYAO NAKANISHI ${ }^{2,4}$, MAYUMI YOSHIMURA $^{2}$, SEIJI ITO $^{5}$, \\ YUKIHIRO SAKAO ${ }^{6}$, YASUHIRO KODERA ${ }^{4}$, YASUSHI YATABE $^{2}$ and NORIO KANEDA ${ }^{1}$ \\ ${ }^{1}$ Laboratory of Analytical Neurobiology, Faculty of Pharmacy, Meijo University, Nagoya, Japan; \\ ${ }^{2}$ Department of Pathology and Molecular Diagnostics, Aichi Cancer Center Hospital, Nagoya, Japan; \\ ${ }^{3}$ Department of Pharmacy, Chubu Rousai Hospital, Nagoya, Japan; \\ ${ }^{4}$ Department of Gastroenterological Surgery, Graduate School of Medicine, Nagoya University, Nagoya, Japan; \\ ${ }^{5}$ Department of Gastroenterological Surgery, Aichi Cancer Center Hospital, Nagoya, Japan; \\ ${ }^{6}$ Department of Thoracic Surgery, Faculty of Medical Technologies, Teikyo Medical University, Tokyo, Japan
}

\begin{abstract}
Background/Aim: Dynamics of circulating tumor cells (CTCs) after molecular targeting therapy remain unclear. Materials and Methods: We examined changes in CTC numbers and morphology early after targeting therapy in EGFR-mutated PC-9 human lung cancer and HER2-gene amplified GLM-1 gastric cancer mouse CTC models using a cytology-based semi-automated CTC detection platform. Results: Erlotinib and T-DM1 inhibited cell growth mainly by induction of apoptosis in vitro. The number of CTCs detected 5-10 days after targeting therapy in mice was significantly increased compared to CTC numbers before therapy. The increased CTCs after therapy consisted of apoptotic CTCs and viable CTCs. This heterogeneous population of CTCs reflects well the cell population of the primary tumor disrupted by therapy. Conclusion: CTCs can be mobilized from the primary tumor due to tissue disruption in acute response to targeting therapy, suggesting potential usefulness of CTC monitoring as a predictor of therapeutic response in the clinical settings.
\end{abstract}

Circulating tumor cells (CTCs) from peripheral blood can be used as a potentially alternative liquid biopsy for the biopsy of metastatic organs (1-3). Recently, cell-free circulating tumor

Correspondence to: Hayao Nakanishi, MD \& PhD, Aichi Cancer Center Hospital, Department of Pathology and Molecular Diagnostics, 1-1 Kanokoden, Chikusa-ku, Nagoya, 464-8681, Japan. Tel: +81 527626111, e-mail: hnakanis@aichi-cc.jp

Key Words: CTC, in vivo dynamics, cytology, lung cancer, gastric cancer, molecular targeting therapy, preclinical study, automated microfluidic device.
DNA (ctDNA) has emerged as a new form of liquid biopsy that enables the detection of genetic alterations in advanced-cancer patients; however, the efficacy of ctDNA as an early diagnostic and predictive marker for recurrence and metastasis remains unclear (4). To date, clinical evidence has demonstrated the usefulness of CTCs in patients with breast, colorectal, lung and prostatic cancers (5). The number of CTCs is a good indicator for patient survival as well as various imaging techniques in localized and metastatic cancer patients (6). The protein and gene profile of CTCs was reported to correlate with prognosis in metastatic breast cancer patients (7). Although evidence has increased for the prognostic significance of CTCs in cancer patients, the clinical significance of CTCs as a predictor for drug sensitivity or resistance and as a monitoring tool for therapeutic effect has not been sufficiently explored $(8,9)$. Basic aspects of the dynamics of CTCs in response to drug therapy also remain unclear (10).

Molecular targeting therapy including various types of tyrosine kinase inhibitors (TKI) and antibody therapy is now the most rapidly growing therapeutic field (11). Lung cancers harboring EGFR mutations such as L858R and Exon 19 deletion, which are major driver gene mutations, are highly sensitive to gefitinib, erlotinib and afatinib, which are reversible and irreversible TKIs (12). These TKIs are currently used as a first-line treatment modality against recurrent or metastatic non-small cell lung cancer (13). Trastuzumab and T-DM1, a trastuzumab-derivative of maytansine 1 conjugate, are now used worldwide as a standard therapy in patients with HER2-positive breast cancer and partly in HER2-positive gastric cancer patients in an adjuvant and neo adjuvant settings $(14,15)$.

Several investigators have previously reported alterations in CTC number after chemotherapy and molecular targeting 
therapy. Most of these reports showed a reduction in CTCs in patients showing high therapeutic efficacy (16-18). Recently, however, Martin et al. reported that CTC number increased early after radiation therapy in lung cancer patients, indicating that CTCs can be mobilized to peripheral blood by radiation therapy due to drug-induced disruption of the primary tumor tissue (19-20). More recently, we demonstrated that CTCs were increased during the early period after chemotherapy with paclitaxel and doxorubicin in mouse breast cancer CTC models, which suggested that CTCs can be mobilized from the primary tumor in rapid response to chemotherapy in addition to radiation therapy $(10)$.

We recently developed a cytology-based CTC detection microfluidic platform using a 3D metal filter that can easily enumerate CTCs and also estimate morphology of CTCs on a glass slide by Papanicolaou (Pap) staining and immunocytochemistry (ICC) under light microscopy (21-23). To more efficiently examine CTC changes in response to molecular targeting therapy, in this study, we improved a cytology-based CTC detection platform to a semiautomated type and used it to investigate CTC dynamics after two types of molecular targeting therapies in preclinical mouse CTC models derived from human lung cancer and gastric cancer cells.

\section{Materials and Methods}

Reagents. Rabbit polyclonal antibody to wide spectrum human cytokeratin (Ab9377) and mouse monoclonal antibodies to human HER2 (Ab16901) were purchased from Abcam (Cambridge, UK). For direct labeling of antibody for immunofluorescence (IF), the Zenon Alexa fluor-488 (-568) mouse labeling kit from Invitrogen (Molecular Probes, Eugene, OR, USA) was used. For counterstaining of nucleus, Hoechst 33342 (Molecular Probes) was used. Erlotinib for in vitro analysis was purchased from Santa Cruz Biotech (Dallas, TX, USA). Erlotinib (Tarceva) for the mouse experiments was obtained from Chugai Pharmaceutical (Tokyo, Japan). T-DM1 was also purchased from Chugai Pharmaceutical.

Cell lines. The PC-9 cell line, an EGFR-mutated human lung cancer cell line, was purchased from ATCC (Manassas, VA, USA). The GLM-1 cell line, a HER2 gene amplified human gastric cancer cell line, was established in our laboratory (24). These cell lines were cultured in Dulbecco's modified Eagle's medium (DMEM) (Nissui Co., Tokyo, Japan) with $10 \%$ fetal bovine serum (FBS) (GIBCO, Grand Island, NY, USA) and penicillin/streptomycin sulfate (SigmaAldrich, St. Louis, MO, USA) in a humidified $5 \% \mathrm{CO}_{2}$ incubator at $37^{\circ} \mathrm{C}$.

Cell growth inhibition assay. PC-9 and GLM-1 cells were harvested with trypsin/EDTA, plated at $1 \times 10^{4}$ cells/well in a 96-well plate in DMEM with $10 \%$ FBS, and cultured for $24 \mathrm{~h}$. Cells were treated with increasing doses of erlotinib $(0.1,1.0,10 \mu \mathrm{M})$ or T-DM1 $(0.1$, $1.0,10 \mu \mathrm{g} / \mathrm{ml}$ ) for several days. Cell number was counted with a hemocytometer in triplicate. Morphology of cells was also examined and photographed by a phase contrast microscope.
Apoptosis assay. Apoptosis was examined using the M30 CytoDEATH staining kit (Roche, Mannheim, Germany) as described previously (25). Cells were plated in $6 \mathrm{~cm}$ plastic plates, cultured for $24 \mathrm{~h}$ in growth medium and treated with erlotinib $(10 \mu \mathrm{M})$ and $\mathrm{T}-$ DM1 $(10 \mu \mathrm{g} / \mathrm{ml})$ for 2-3 days. Cells were stained for M30 and observed under light microscopy (Eclipse Ti-S, Nikon, Tokyo, Japan).

Immunofluorescence (IF) staining and fluorescence in situ hybridization (FISH) Analysis. CTC specimens on a slide glass were stained by IF using Alexa 488-labeled mouse anti-human HER2 antibody. Amplification of the HER2 gene was assessed by a dualcolor FISH method using a Passvision HER-2 DNA Probe Kit (Vysis Inc., IL, USA) according to the manufacturer's protocol. The HER-2/neu-Spectrum Orange probe identifies the human HER2 gene locus in chromosome 17q11.2-q12. The CEP 17 green probe recognizes the centromere region of chromosome 17 as a control. The nucleus was stained with DAPI. The slides were observed under a BX60 fluorescence microscope with a digital camera (DP50, Olympus, Tokyo, Japan). Cells with more than four orange signals for HER2 per cell were considered as HER2 amplification.

Detection of EGFR mutation. Tumor cells on a glass slide were dissolved by incubation with proteinase $\mathrm{K}$ solution for $1 \mathrm{~h}$ at $54^{\circ} \mathrm{C}$. After heat inactivation at $95^{\circ} \mathrm{C}$, the solution was directly used as template DNA for mutation analysis. To detect exon 19 deletion in the $E G F R$ gene, fragment analysis was performed as described previously (26). Briefly, PCR products were electrophoresed on an ABI PRISM 310. Deletion mutation analysis was conducted by the detection of the shorter fragment DNA as a new peak in an electropherogram. In PC9 cells, a 15-bp deletion of exon 19 was detected.

Animals. Seven- to nine-week-old female athymic nude mice (KSN strain, 27-30 g) were obtained from Japan SLC (Hamamatsu, Japan). Mice were maintained and used under specific pathogenfree (SPF) conditions. All animal experiments were performed according to the experiment protocol approved by the Ethics Review Committee for Animal Experimentation of the Aichi Cancer Center and met the standard as defined by the recently reported international guidelines (27).

Filtration-based CTC isolation and transfer to glass slides. The CTC detection procedure consists of the following three steps: 1) enrichment of CTCs by a semiautomated CTC isolation apparatus including a microfluidic device with 3-dimensional (3D) nickel filter (Optnics Precision Co., Tochigi, Japan); 2) transfer of CTCs from the 3D metal filter to a glass slide (CTC glass slide); and 3) cytological examination of the CTC glass slide by Papanicolaou (Pap) staining and immunocytochemistry (ICC). A semiautomated CTC isolation apparatus with a touch panel improved in this study consists of peristaltic pumps, a diaphragm pump, an electromagnetic valve, and optical liquid level sensors regulated by a programmable logic controller (Figure 1A). Procedures for CTC detection were essentially the same as previously described (10): blood (0.1-0.25 ml) from the mouse heart was diluted 10-fold with PBS with $0.5 \mathrm{mM}$ EDTA (PBS/EDTA) and then filtrated with a semiautomated isolation device at a flow rate of $7 \mathrm{ml} / \mathrm{min}$. After filtration of blood, cells trapped in a filter were fixed with $10 \%$ formalin for $30 \mathrm{~min}$ and briefly washed with PBS/EDTA in the device. The filter detached from the device was placed upside down onto a glass slide (MAS coat, Matsunami, Osaka, Japan) and immersed with PBS/EDTA. The CTCs trapped in 
A Filtration device Semiautomated CTC enrichment device

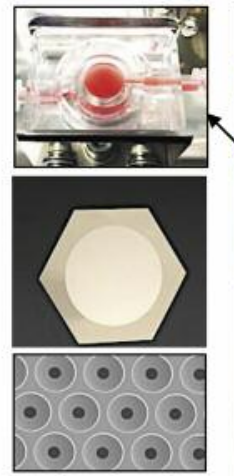

3D metal Filter

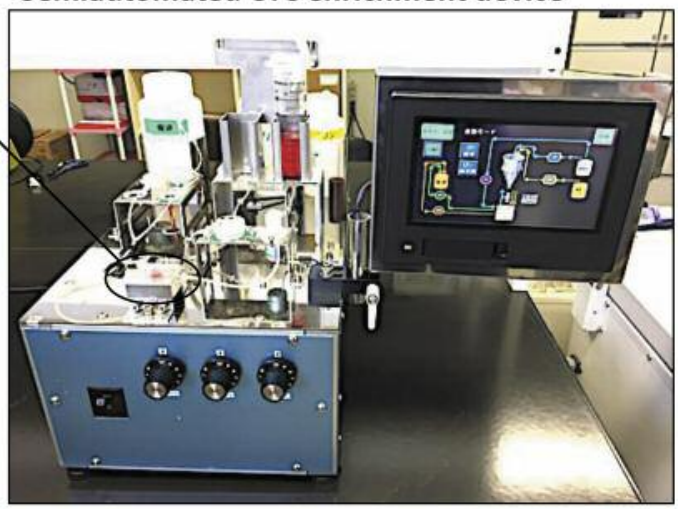

B

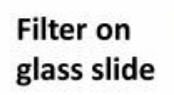

glass slide

CTC transfer

to glass

slide by

pressure

device
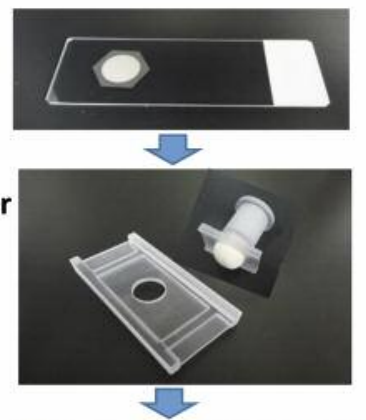

CTC glass

slide

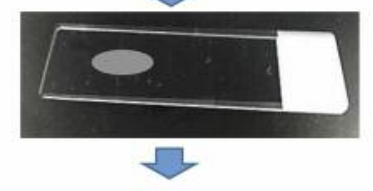

Staining

C
Pap/ICC

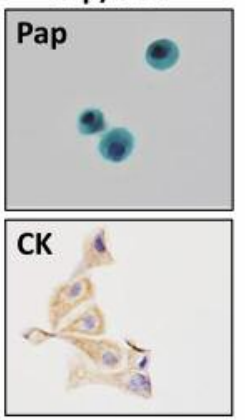

Pap/triple IF

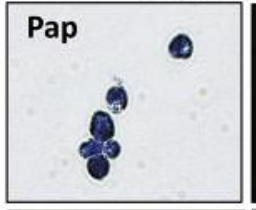

CD45

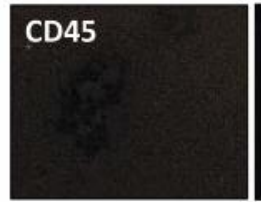

CK

Q
0-1 M

1-2 M

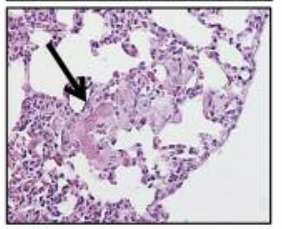

$0-1 M(n=5) \quad 1-2 M(n=8)$

Figure 1. Overview of the cytology-based CTC detection platform and lung cancer mouse CTC model. A: Filtration device with $3 D$ metal filter (pore size: $8 \mu \mathrm{m}$ ) and semiautomated CTC enrichment apparatus. B: Transfer of CTCs to a glass slide by a handheld mild pressure device and a resultant CTC glass slide. C: CTCs stained with cytology (Pap) and immunocytochemistry (ICC) (left) and CTCs stained by cytology (Pap) and CK/CD45/Hoechst triple immunofluorescence (IF) (right). CK: Cytokeratin; D: Lung cancer mouse CTC model bearing subcutaneous (sc) tumors formed after injection of PC-9 lung cancer cells into nude mice. Representative metastasis in the lung at 1-2 months (M) after sc injection revealed by Hematoxylin-Eosin (HE) staining. Arrow indicates micrometastasis in the lung (left). Number of CTCs in blood according to time (M) after sc injection of PC-9 cells (right).

the filter were then quickly transferred to a glass slide using a handheld mild mechanical pressure device with sponge at the tip (Optnics Precision Co., Tochigi, Japan) (Figure 1B). The resultant CTCs attached to the glass slide were immediately fixed in $95 \%$ ethanol for Pap staining or fixed in $95 \%$ ethanol, followed by $10 \%$ buffered formalin for ICC.

Cytological and ICC staining of CTCs. Pap staining of a glass specimen was conducted by an automatic stainer (Sakura Fintec, Tokyo, Japan). For immunostaining, after blocking with 1\% BSA, the specimen was incubated with rabbit antibodies to pancytokeratin for $2 \mathrm{~h}$. After washing, the specimens were incubated with HRP-labeled polymer conjugated with goat anti-rabbit antibody (EnVision+System, DAKO) for $30 \mathrm{~min}$. After washing with PBS, the chromogen was developed with the Liquid $\mathrm{DAB}+$ substrate chromogen system (DAKO). The nuclei were stained with Meyer's hematoxylin.
Mouse CTC models and treatment strategies. Cultured PC-9 or GLM-1 cells $\left(5 \times 10^{6}\right)$ suspended in Hank's balanced salt solution were subcutaneously $(\mathrm{sc})$ injected into the back region of mice. To evaluate the anti-tumor effect of the targeting drugs, mice with PC9 transplanted tumors were orally administered erlotinib (100 $\mathrm{mg} / \mathrm{kg} /$ day, from day 7 post-injection, 5 times per week for 5 weeks) by gastric tube. Control mice were orally administered vehicle $(0.5 \%$ polysorbate in distilled water). In mice with GLM-1 cellderived tumors, the tumors were intraperitoneally (ip) injected with T-DM1 in saline ( $40 \mathrm{mg} / \mathrm{kg} / \mathrm{day}$, once a week for 4 weeks). Control mice were administered vehicle (saline). Maximum tumor diameter (L) and short diameter (W) were measured every week and tumor volume was estimated by the following formula: $\mathrm{L} \times \mathrm{W} \times \mathrm{W} \times 1 / 2$.

To monitor CTC dynamics after targeting therapy, mice bearing tumor with 1-2 cm diameter approximately 2 months post-injection were used. Blood was harvested by cardiac puncture in a tube with EDTA before and after targeting therapy to tumor bearing mouse. 
A

$$
\text { PC-9 cells }
$$

Deletion in exon 19 of EGFR gene

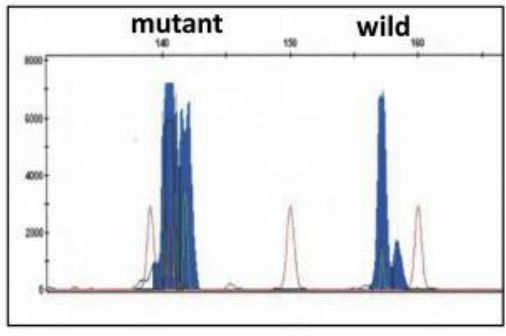

B

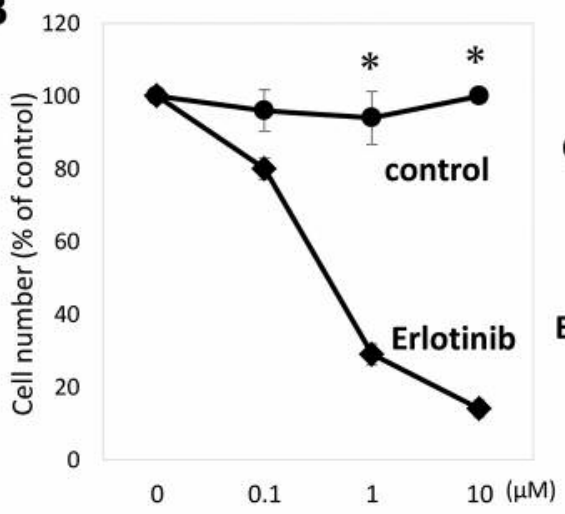

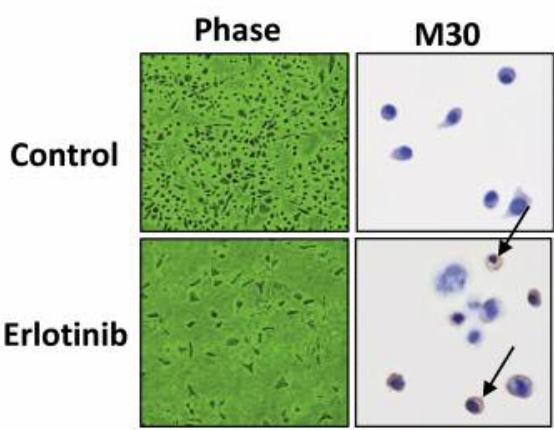

C

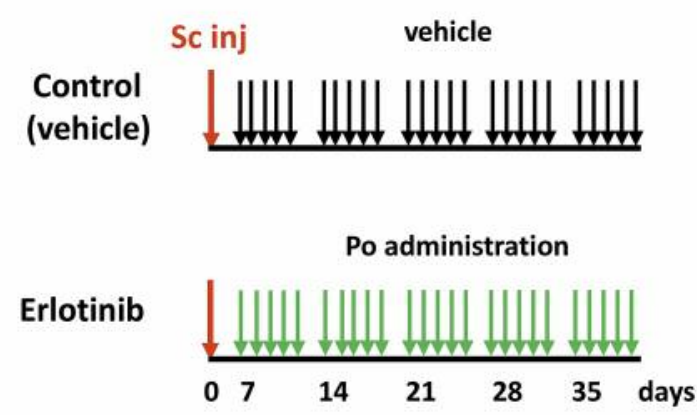

D

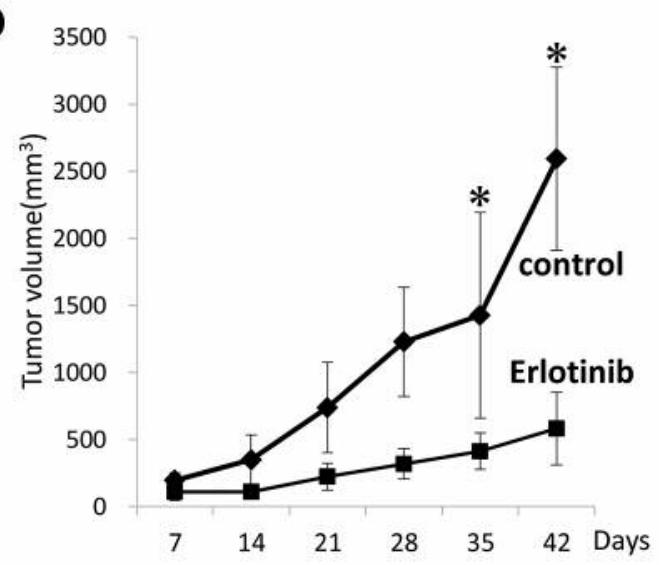

Figure 2. In vitro and in vivo growth inhibition of PC-9 lung cancer cells by erlotinib. A: A 15-bp deletion in exon 19 in the EGFR gene demonstrated by common fragment analysis. B: Effects of erlotinib on the growth of PC-9 cells (left) as well as morphology and M30 staining (right) in culture. Cells in 96-well dishes were treated with erlotinib $(0-10 \mu \mathrm{M})$ for $48 \mathrm{~h}$, followed by cell counting and M30 immunostaining. Arrows indicate M30-positive apoptotic cells. C: Treatment strategy of tumor bearing mice ( $n=5)$. Erlotinib was orally administered (100 mg/kg/day, 5 times/week for 5 weeks) in the PC-9 cell mouse CTC model. Anti-tumor effects of erlotinib on subcutaneous (sc) tumor growth in mice. ${ }^{*} p<0.01$ vs. vehicle control. Bars=SD.

As a preliminary experiment, we compared routes of serial blood collection for CTCs such as the lateral tail vein, retroorbital venous plexus and the heart. As a result, cardiac puncture from the heart was found to be the only route available in terms of safety, repetitiveness and less contamination risk for normal epithelial cells. Frequency and volume of blood sampling used in our study was based on the body weight and interval period, as recommended by the guidelines $(28,29)$. Cardiac puncture of mice was safely performed using a $27 \mathrm{G}$ syringe under 2, 2, 2-tribromoethanol anesthesia without significant side effect. Briefly, we collected approximately $0.20 \mathrm{ml}$ blood before and 5-10 days after targeting therapy with erlotinib (oral administration, $100 \mathrm{mg} / \mathrm{kg} /$ day, 5 times) or T-DM1(ip injection, $40 \mathrm{mg} / \mathrm{kg} / \mathrm{day}$, once) and in some cases further collected at several weeks after treatment. After the experiment, mice were euthanatized and primary subcutaneous tumor and lung metastases were examined by histological examination with hematoxylin-eosin (HE) staining.

Statistical analysis. The statistical significance of differences in data between groups was determined by Student's $t$-test. A $p$-value $<0.05$ was considered significant. A p-value of 0.1-0.05 was considered marginally significant.

\section{Results}

Cytology-based CTC detection method and CTC models in mice. The procedure for our cytology-based CTC detection system involved the following steps: 1) isolation of CTCs by the semiautomated filtration device (Figure 1A), 2) CTC transfer from filter to a glass slide by a gentle pressure device (Figure 1B), and 3) cytological examination of CTCs by Pap and ICC staining (Figure 1C, left). Triple IF staining of CTC specimens using Alexa 488-labeled anti-human cytokeratin and PE-labeled anti-mouse CD45 showed that atypical cells stained by Pap were CK-positive and CD45-negative, confirming that $\mathrm{CK}$-positive cells with cytologically atypical morphology can be correctly judged as CTCs under light microscopy (Figure 1C, right). 
A
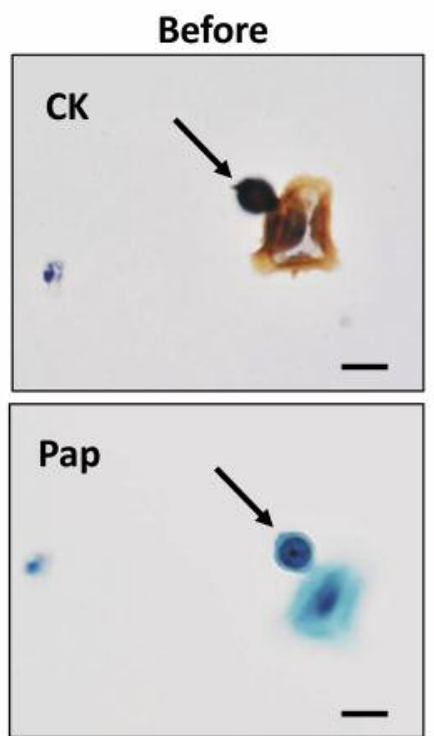

C

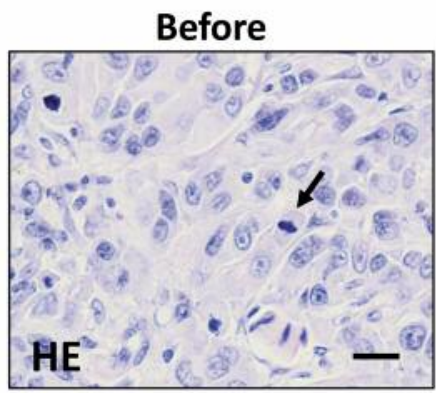

After therapy
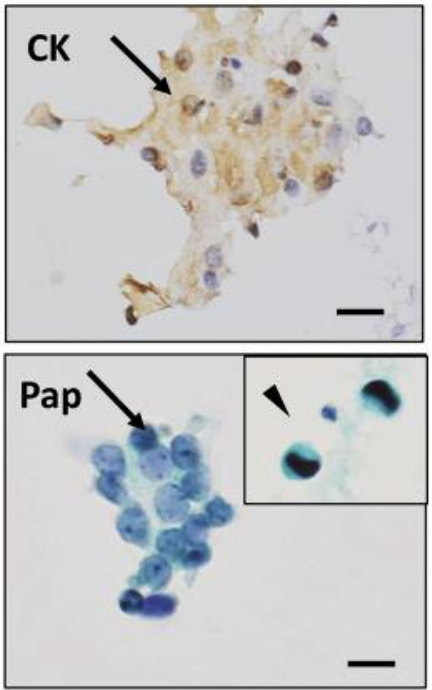

After therapy

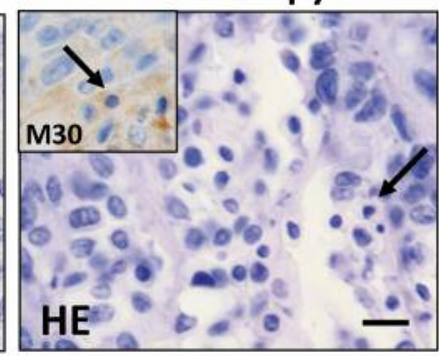

B
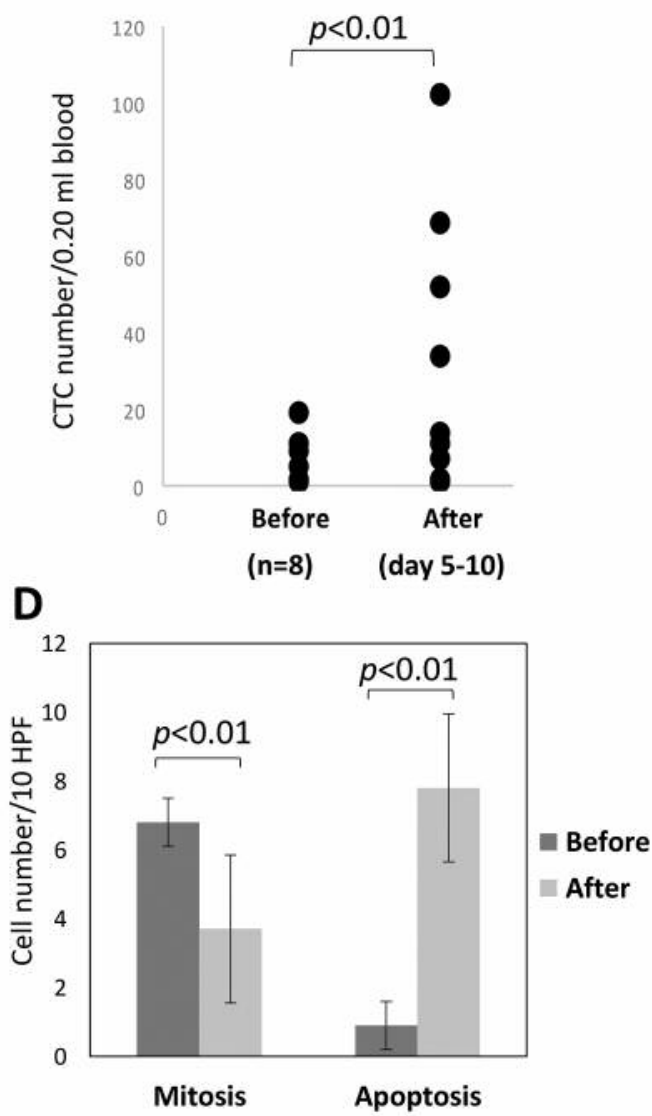

Figure 3. Changes in the number and morphology of CTCs after erlotinib treatment in PC-9 tumor-bearing mice. A: Cytokeratin (CK)-positive CTCs in blood before and 5-10 days after erlotinib treatment (top). CTCs stained by Papanicolaou (Pap) in blood before and after erlotinib treatment (bottom). Arrows and arrow head indicate healthy CTCs and apoptotic CTCs (inset), respectively. B: Significant increase in CTC number 5-10 days after erlotinib treatment compared with before treatment in PC-9 CTC model $(n=8)(p<0.01)$. C: Histology of the primary sc tumors before and 510 days after erlotinib treatment as shown by HE staining. Mitotic tumor cells (left) and apoptotic tumor cells (right) are indicated by arrows. Apoptotic cells are stained positive for M30 (inset). Bar $=20 \mu \mathrm{m}$. D: Comparison of the numbers of mitotic and apoptotic tumor cells (number of specified cells/10 high power fields $=H P F)$ in the primary tumor tissue before and after erlotinib treatment $(n=5)$.

We next developed mouse CTC models using human lung cancer cells (PC-9) and gastric cancer cells (GLM-1). These models are formed by subcutaneous injection of PC-9 cells with spontaneous metastatic potential into nude mice. Lung metastasis was visualized approximately 1-2 months after subcutaneous injection (Figure 1D, left). A significantly higher number of CTCs was detected in mise with metastasis $(p<0.05)$ (Figure 1D, right).

Growth inhibition by erlotinib in vitro and in the mouse CTC model. PC-9 cells were confirmed to have exon 19 deletion of the EGFR gene by fragment length analysis (Figure 2A). Therefore, PC-9 cells are presumed to be sensitive to erlotinib. We confirmed that the growth of PC-9 cells in vitro was significantly inhibited by erlotinib in a dose-dependent manner $(p<0.01)$. In cells treated with erlotinib $(10 \mu \mathrm{M})$ for $24 \mathrm{~h}$, abundant small floating cells were observed. M30 cytodeath staining confirmed the presence of apoptotic tumor cells (Figure 2B). In addition, erlotinib treatment of the PC9 subcutaneous tumor bearing mice resulted in significant anti-tumor effects compared with control treatment with vehicle $(p<0.01)$ (Figure 2C and D).

Changes in CTCs before and after erlotinib treatment in the mouse CTC model. We first examined CTCs from mice before erlotinib treatment based on the following criteria: keratinpositive cells with atypical nucleus as revealed by ICC with hematoxylin counter staining. CTCs were observed in single 
A

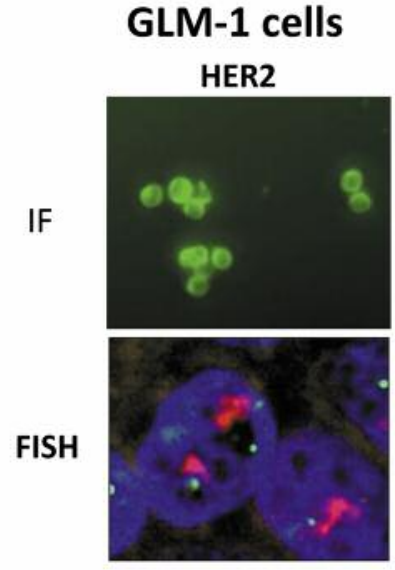

C

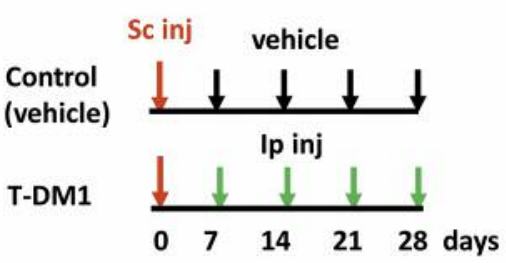

B

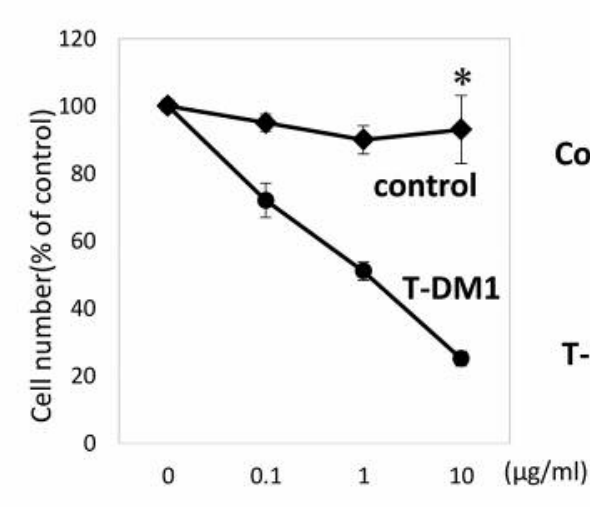

M30

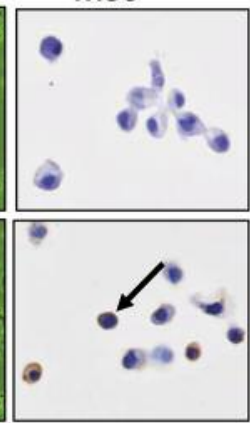

D

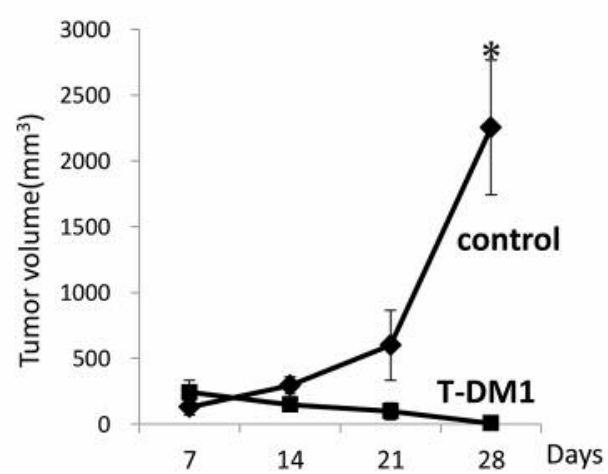

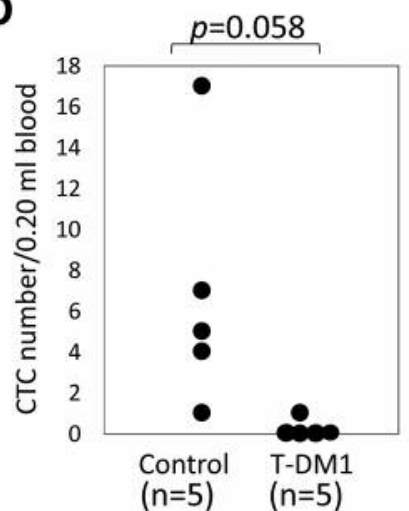

Figure 4. In vitro and in vivo growth inhibition of HER2-gene amplified GLM-1 gastric cancer cells by T-DM1. A: HER2 overexpression and cluster type gene amplification in GLM-1 cells was detected by IF and FISH analysis, respectively. B: Growth inhibition of GLM-1 cells by increasing dose of T-DM1 in culture. * ${ }^{p}<0.01$ vs. vehicle control. Bars=SE. Effects of T-DM1 on morphology and apoptosis induction (M30 staining) in GLM-1 cells. Cells plated in a 96-well dish were treated with T-DM1 $(0-10 \mu \mathrm{g} / \mathrm{ml})$ for 3 days, followed by cell counting and M30 staining. Arrows indicate M30-positive apoptotic cells. C: Treatment strategy of tumor bearing mice ( $\mathrm{n=5}$ ). T-DM1 was administered (40 mg/kg mouse/day, weekly, for 4 weeks) by intraperitoneal (ip) injection into GLM-1 tumor-bearing mice (left). Effect of T-DM1 on the subcutaneous tumor growth in mice was examined based on tumor volume. ${ }^{*} p<0.01$ vs. vehicle control. Bars $=S D$. D: Decrease in CTC number with marginal significance ( $\left.p=0.058\right)$ after completion of T-DM1 treatment compared with before treatment in the G-LM1 cell tumor-bearing mice ( $n=5)$.

cell and cluster patterns (Figure 3A). The number of CTCs was significantly increased at 5-10 days after erlotinib treatment compared with before treatment $(p<0.01)$ (Figure 3B). Pap staining showed that these increased CTCs included both small apoptotic CTCs with condensed nuclei and CTCs with healthy morphology (Figure 3A). Histological analysis of the primary subcutaneous tumors demonstrated an increase in the popularity of apoptotic tumor cells after treatment (Figure 3C). Quantitative analysis of the primary tumor showed that the number of mitotic tumor cells in the primary tumor tissue significantly decreased 5-10 days after erlotinib treatment compared with those before treatment $(p<0.01)$. In contrast, apoptotic tumor cells in the primary tumor significantly increased 5-10 days after erlotinib treatment $(p<0.01)$ (Figure 3D). The appearance of apoptotic CTCs in the blood early after treatment seemed to correspond to the increase in apoptotic tumor cells in the primary tumor.

Because of ethical and technical reasons of cardiac puncture, the examination of sequential changes in CTC number at various times (before treatment, 5-10 days after treatment and 20-30 days after treatment) is difficult, but some preliminary data from 2 mice showed that the numbers of CTCs increased at 5 days post-injection and then decreased to baseline at 25 days post-injection (data not shown). This suggests the possibility that the increase in CTC number after treatment may be transient and an acute response to the treatment. 
A
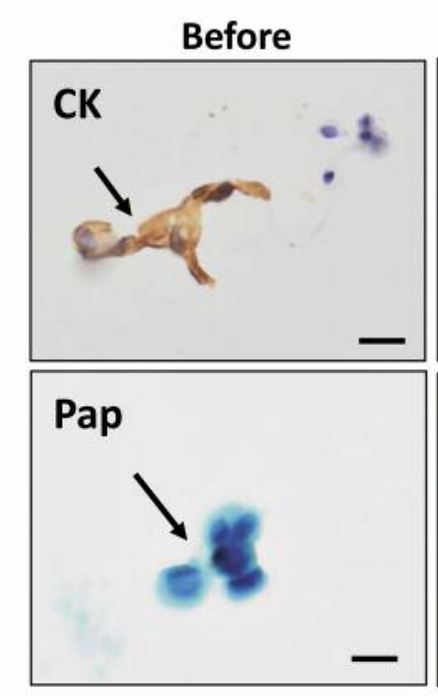

C

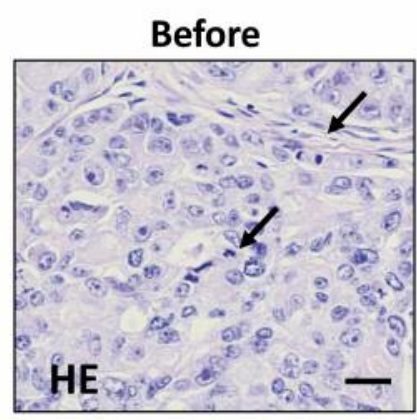

After therapy
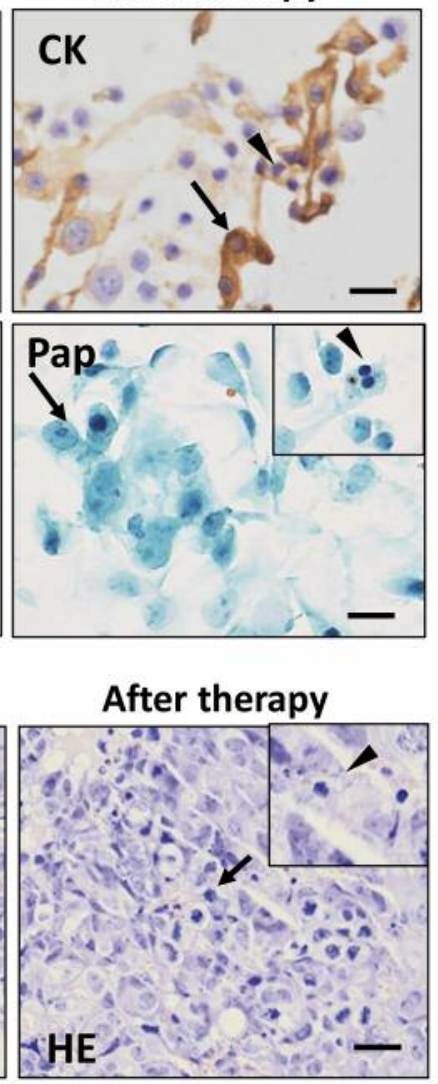

B

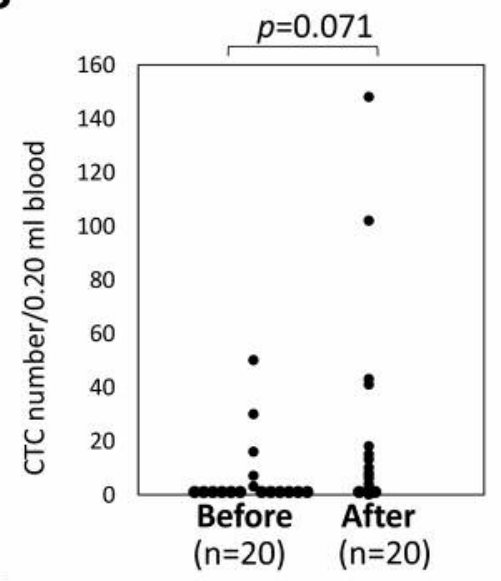

D

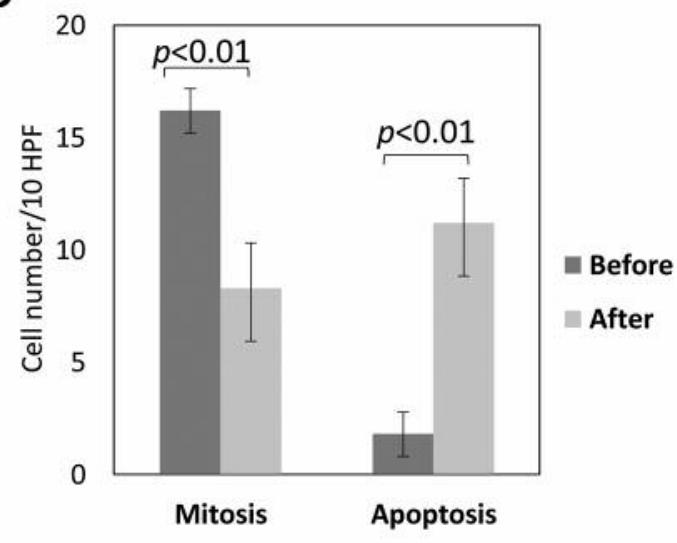

Figure 5. Changes in the number and morphology of CTCs after T-DM1 treatment in a GLM-1 mouse CTC model. A: Cytokeratin (CK)-positive CTCs detected in blood before and 5-10 days after T-DM1 treatment (top). CTCs stained by Pap before and after T-DM1 treatment (bottom). Arrows and arrowheads indicate CTCs and apoptotic CTCs, respectively. B: Increase in CTC number 5-10 days after T-DM1 treatment compared with before treatment in GLM-1 tumor-bearing mice $(n=20)$ with marginal significance $(p=0.071)$. C: Histology of the primary subcutaneous tumors in mice before and 5-10 days after T-DM1 treatment, as determined by HE staining. Mitotic tumor cells (before) and M-phase arrested tumor cells (after T-DM1) are indicated by arrows. Arrowhead (inset) showed apoptotic CTC. Bar=20 $\mu \mathrm{m}$. D: Comparison of the numbers of mitotic and apoptotic tumor cells (number of specified cells/10 high power fields=HPF) before and after T-DM1 treatment in the primary tumor tissue (n=5).

Growth inhibition by T-DM1 in vitro and in the mouse CTC model. GLM-1 cells showed high HER2 protein expression by IF and cluster type HER2-gene amplification by FISH analysis (Figure 4A). The growth of GLM-1 cells was significantly inhibited by T-DM1 in a dose-dependent manner $(p<0.01$; Figure 4B). In cells treated with T-DM1 $(10 \mu \mathrm{g} / \mathrm{ml})$ for 3 days, abundant floating small cells were observed. M30 cytodeath staining confirmed the presence of many apoptotic tumor cells (Figure 4B). In mice bearing subcutaneous tumors from GLM-1 cells, T-DM1 treatment by intraperitoneal injection exhibited significant anti-tumor activity compared with control treatment with vehicle $(p<0.01)$ (Figure 4C). The number of CTCs examined after completion of treatment for 4 weeks was decreased compared with vehicle control with marginal significance $(p=0.058$, Figure 4D).

Changes in CTCs before and after T-DM1 treatment in the mouse CTC model. T-DM1 treatment in the GLM-1 mouse model showed that the number of CK-positive CTCs tended to increase at 5-10 days post-injection compared with CTC numbers before treatment, with marginal significance $(p=0.071)$. The increased CTCs detected after T-DM1 therapy consists of apoptotic cells and morphologically healthy cells (Figure 5A and B). Histological analysis of primary sc tumors demonstrated increased numbers of apoptotic tumor cells $(p<0.01)$ and decreased numbers of mitotic tumor cells 5-10 days after injection of T-DM1 
compared with numbers before treatment $(p<0.01)$ (Figure $5 \mathrm{C}$ and $\mathrm{D})$.

\section{Discussion}

Previous studies demonstrated that the number of CTCs in blood decrease at a late stage after effective anti-tumor therapy $(8,9)$. However, Martin et al. recently reported a transient increase in CTC number as a result of CTC mobilization from the primary tumor, early after radiation therapy in clinical cancer patients $(19,20)$. Furthermore, we examined acute phase dynamics of CTCs after chemotherapy in two preclinical breast cancer mouse models and demonstrated that the CTC number transiently increased early after chemotherapy with paclitaxel and doxorubicin (10). In the present study, we investigated whether such acute-phase CTC dynamics are also observed early after molecular targeting therapy such as TKI in lung cancer mouse CTC models. We found that the number of CTCs significantly increased early (5-10 days) after erlotinib treatment in EGFR-mutated PC-9 lung cancer xenograft tumors in mice. We also found that the increased CTC population consisted of both apoptotic CTCs and viable CTCs with healthy morphology. Furthermore, even though the numbers of examined mice were small $(n=2)$ in this study as the result of ethical and technical reasons, we found that CTCs tended to decrease to baseline levels during the late period (2-3 weeks) after therapy, suggesting that CTCs transiently increased early (5-10 days) after erlotinib therapy. Histological analysis of the primary tumors at 5-10 days after erlotinib treatment revealed abundant apoptotic tumor cells and viable tumor cells in the primary subcutaneous tumor, which corresponds to the increase in apoptotic CTCs together with intact CTCs after erlotinib therapy. These findings suggest that CTCs can be mobilized from the primary tumor into blood circulation due to tissue disruption derived from the anti-tumor effects of molecular targeting therapy. To the best of our knowledge, the present study is the first report indicating the mobilization of CTCs early after targeting therapy with TKI in a lung cancer preclinical model.

To examine the generality of the increase in CTC number early after targeting therapy, we further examined changes in CTCs in mice treated with another targeting therapy, T-DM1, using the HER2-positive gastric cancer mouse CTC model. We found that CTCs also tended to increase 5-10 days after T-DM1 treatment $(p=0.07)$. The CTC population detected after T-DM1 treatment consisted of apoptotic CTCs, M-phase arrested CTCs and viable CTCs with healthy morphology. Mphase-arrested CTCs may be due to inhibition of microtubule formation by antibody-conjugated maytansine, a microtubule polymerization inhibitor (30). Such heterogeneity of CTCs is similar to the tumor cell population observed in the primary tumors exposed to T-DM1 treatment. These results indicate that CTC number increased in early response to molecular targeting therapies with both TKI (erlotinib) and antibody (TDM1) and suggest that mobilization of affected tumor cells from the disrupted primary tumor to the blood occurs in the acute response to molecular targeting therapies as well as to chemotherapy (10).

The mechanism underlying the increase in CTC number early after targeting therapy remains unclear. One possible explanation is the enhanced mobilization of apoptotic CTCs together with viable CTCs from the primary tumor due to passive intravasation of tumor cells into blood vessels as the result of destruction of tumor cell-cell interactions and disruption of tumor blood vessels (20). Another explanation is the involvement of epithelial-mesenchymal transition (EMT) induced by an EGFR TKI such as gefitinib (31). EMT can enhance the motility or migratory activity of tumor cells, leading to active cell intravasation into blood vessels. The antibody used in this study recognizes a wide spectrum of human cytokeratins that is potentially detectable epithelial tumor cells with some EMT phenotype. The presence of CTCs with an EMT phenotype has also been previously reported in a clinical study of breast cancer patients under treatment with targeting therapy (32). Irrespective of the underlying mechanisms, mobilization of non-apoptotic, morphologically viable CTCs into systemic blood circulation during the early phase after targeting therapy suggests a potential risk for metastasis to distant organs such as the lung. This possibility is consistent with the recent report by Martin et al. that CTC mobilization after radiation therapy and other anti-cancer therapy might promote hematogenous metastasis and therefore, be associated with an unfavorable patient outcome (20).

Unique features of the newly developed cytology-based semiautomated CTC detection system contributed a lot to the new findings of this study. Most previous CTC detection methods have been based on immunofluorescence criteria such as $\mathrm{Keratin}^{+} / \mathrm{EpCAM}^{+} / \mathrm{CD} 45^{-} / \mathrm{DAPI}^{+}$expression under dark field $(1,2)$. The difficulty with such an immunofluorescence approach under dark field is the insufficient morphological and cytological estimation of CTCs, especially damaged CTCs such as apoptotic, M-phase arrested and degenerated CTCs, generated by anti-cancer therapies (33). Our cytology-based CTC detection system overcomes this problem by the staining of CTC glass slides by Pap and ICC and the subsequent cytological diagnosis by a cytopathologist using permanent CTC specimens under light microscopy $(22,23)$ which is a different method from the previous report (34). We also improved the previous manual device to a semiautomated one in this study that enables more rapid and convenient CTC enrichment, leading to a more practical cytology-based CTC detection platform.

In conclusion, using a currently developed cytology-based semiautomated CTC detection platform, we demonstrated the increase in the CTC number in acute response to molecular targeting therapies with both TKI and antibody therapy. The 
heterogeneous population of CTCs mobilized after targeting therapy reflects the anti-tumor effect observed in the primary tumor by the therapy. Therefore, sequential monitoring of CTCs in terms of number and morphology using our costeffective cytology-based CTC detection platform from the early stage of targeting therapy, would be a powerful strategy to predict response of the primary tumor to drug therapy.

\section{Conflicts of Interest}

The Authors have no conflicts of interest regarding this study.

\section{Authors' Contributions}

A.I., H.N., and N.K. contributed to the study conception and design. A.I., and M.Y. performed in vitro experiments. A.I., and H.N. performed in vivo experiment using mouse CTC models. A.I., and M.Y. performed CTC isolation and their cytological examination with staining. Y.Y. performed mutation and FISH analysis. A.I., and H.N. drafted the manuscript. S.I., Y.S., and Y.K. contributed to the interpretation of the results and provided the critical feedback.

\section{Acknowledgements}

This study was supported in part by a Grant-in-Aid for Ministry of Education, Science, Sports, Culture and Technology, Japan. The Authors thank Edanz Gr (www.edanzediting.com/ac) for editing a draft of this manuscript.

\section{References}

1 Cristofanilli M, Budd GT, Ellis MJ, Stopeck A, Matera J, Miller MC, Reuben JM, Doyle GV, Allard WJ, Terstappen LW and Hayes DF: Circulating tumor cells, disease progression, and survival in metastatic breast cancer. N Engl J Med 351: 781-791, 2004. PMID: 15317891. DOI: 10.1056/NEJMoa040766

2 Riethdorf S, O'Flaherty L, Hille C and Pantel K: Clinical applications of the CellSearch platform in cancer patients. Adv Drug Deliv Rev 125: 102-121, 2018. PMID: 29355669. DOI: 10.1016/j.addr.2018.01.011

3 Tan $\mathrm{Y}$ and $\mathrm{Wu} \mathrm{H}$ : The significant prognostic value of circulating tumor cells in colorectal cancer: A systematic review and metaanalysis. Curr Probl Cancer 42(1): 95-106, 2018. PMID: 29277243. DOI: 10.1016/j.currproblcancer.2017.11.002

4 Ignatiadis M, Lee M and Jeffrey SS: Tumor cells and circulating tumor DNA: challenges and opportunities on the path to clinical utility. Clin Cancer Res 21(21): 4786-4800, 2015. PMID: 26527805. DOI: 10.1158/1078-0432.CCR-14-1190

5 Hardingham JE, Grover P, Winter M, Hewett PJ, Price TJ and Thierry B: Detection and clinical significance of circulating tumor cells in colorectal cancer-20 years of progress. Mol Med 21: S2531, 2015. PMID: 26605644. DOI: 10.2119/molmed. 2015.00149

6 Budd GT, Cristofanilli M, Ellis MJ, Stopeck A, Borden E, Miller MC, Matera J, Repollet M, Doyle GV, Terstappen LW and Hayes DF: Circulating tumor cells versus imaging-predicting overall survival in metastatic breast cancer. Clin Cancer Res 12(21): 6403-6409, 2006. PMID: 17085652. DOI: 10.1158/10780432.CCR-05-1769
7 Mostert B, Sieuwerts AM, Kraan J, Bolt-de Vries J, van der Spoel P, van Galen A, Peeters DJ, Dirix LY, Seynaeve CM, Jager A, de Jongh FE, Hamberg P, Stouthard JM, Kehrer DF, Look MP, Smid M, Gratama JW, Foekens JA, Martens JW and Sleijfer S: Gene expression profiles in circulating tumor cells to predict prognosis in metastatic breast cancer patients. Ann Oncol 26(3): 510-516, 2015. PMID: 25471333. DOI: 10.1093/annonc/mdu557

8 Hall C, Karhade M, Laubacher B, Anderson A, Kuerer H, DeSynder $S$ and Lucci A: Circulating tumor cells after neoadjuvant chemotherapy in Stage I-III triple-negative breast cancer. Ann Surg Oncol 22: S552-558, 2015. PMID: 25968619. DOI: $10.1245 / \mathrm{s} 10434-015-4600-6$

9 Peeters DJ, Van Dam PJ, Van den Eynden GG, Rutten A, Wuyts H, Pouillon L, Peeters M, Pauwels P, Van Laere SJ, van Dam PA, Vermeulen PB and Dirix LY: Detection and prognostic significance of circulating tumor cells in patients with metastatic breast cancer according to immunohistochemical subtypes. Br J Cancer 110(2): 375-383, 2014. PMID: 24366294. DOI: 10.1038/ bjc. 2013.743

10 Adachi Y, Yoshimura M, Nishida K, Usuki H, Shibata K, Hattori M, Kondo N, Yatabe Y, Iwata H, Kikumori T, Kodera Y and Nakanishi H: Acute phase dynamics of circulating tumor cells after paclitaxel and doxorubicin chemotherapy in breast cancer mouse models. Breast Cancer Res Treat 167: 439-445, 2018. PMID: 29027049. DOI: 10.1007/s10549-017-4532-x

11 Paez JG, Jänne PA, Lee JC, Tracy S, Greulich H, Gabriel S, Herman P, Kaye FJ, Lindeman N, Boggon TJ, Naoki K, Sasaki H, Fujii Y, Eck MJ, Sellers WR, Johnson BE and Meyerson M: EGFR mutations in lung cancer: correlation with clinical response to gefitinib therapy. Science 304(5676): 14197-1500, 2004. PMID: 15118125. DOI: 10.1126/science.1099314

12 Mitsudomi T, Morita S, Yatabe Y, Negoro S, Okamoto I, Tsurutani J, Seto T, Satouchi M, Tada H, Hirashima T, Asami K, Katakami N, Takada M, Yoshioka H, Shibata K, Kudoh S, Shimizu E, Saito H, Toyooka S, Nakagawa K, Fukuoka M and West Japan Oncology Group: Gefitinib versus cisplatin plus docetaxel in patients with non-small cell lung cancer harbouring mutations of the EGFR (WJTOG3405): an open label, randomized phase 3 trial. Lancet Oncol 11(2): 121-128, 2010. PMID: 20022809. DOI: 10.1016/S1470-2045(09)70364-X

13 Park K, Bennouna J, Boyer M, Hida T, Hirsh V, Kato T, Lu S, Mok T, Nakagawa K, O'Byrne K, Paz-Ares L, Schuler M, Sibilot DM, Tan EH, Tanaka H, Wu YL, Yang JC, Zhang L, Zhou C, Märten A, Tang W and Yamamoto N: Sequencing therapy following first-line afatinib in patients with EGFR mutationpositive non-small cell lung cancer. Lung Cancer 132: 126-131, 2019. PMID: 31097085. DOI: 10.1016/j.lungcan.2019.04.014

14 Verma S, Miles D, Gianni L, Krop IE, Welslau M, Baselga J, Pegram M, Oh DY, Diéras V, Guardino E, Fang L, Lu MW, Olsen S, Blackwell K and EMILIA Study Group: Trastuzumab emtansine for HER2-positive advanced breast cancer. N Engl J Med 8369(19): 1783-1791, 2012. PMID: 23020162. DOI: 10.1056/NEJMoa1209124

15 Thuss-Patience PC, Shah MA, Ohtsu A, Van Cutsem E, Ajani JA, Castro H, Mansoor W, Chung HC, Bodoky G, Shitara K, Phillips GDL, van der Horst T, Harle-Yge ML, Althaus BL and Kang YK: Trastuzumab emtansine versus taxane use for previously treated HER2-positive locally advanced or metastatic gastric or gastrooesophageal junction adenocarcinoma (GATSBY): an international 
randomised, open-label, adaptive, phase 2/3 study. Lancet Oncol 18(5): 640-653, 2017. PMID: 28343975. DOI: 10.1016/S14702045(17)30111-0

16 Torphy RJ, Tignanelli CJ, Kamande JW, Moffitt RA, Herrera Loeza SG, Soper SA, and Yeh JJ: Circulating tumor cells as a biomarker of response to treatment in patient-derived xenograft mouse models of pancreatic adenocarcinoma. PLoS One 9(2): e89474, 2014. PMID: 24586805. DOI: 10.1371/journal.pone. 0089474

17 Rossi E, Fassan M, Aieta M, Zilio F, Celadin R, Borin M, Grassi A, Troiani L, Basso U, Barile C, Sava T, Lanza C, Miatello L, Jirillo A, Rugge M, Indraccolo S, Cristofanilli M, Amadori A and Zamarchi R: Dynamic changes of live/apoptotic circulating tumour cells as predictive marker of response to sunitinib in metastatic renal cancer. Br J Cancer 107(8): 1286-1294, 2012. PMID: 22955853. DOI: 10.1038/bjc.2012.388

18 Bidard FC, Peeters DJ, Fehm T, Nolé F, Gisbert-Criado R, Mavroudis D, Grisanti S, Generali D, Garcia-Saenz JA, Stebbing J, Caldas C, Gazzaniga P, Manso L, Zamarchi R, de Lascoiti AF, De Mattos-Arruda L, Ignatiadis M, Lebofsky R, van Laere SJ, Meier-Stiegen F, Sandri MT, Vidal-Martinez J, Politaki E, Consoli F, Bottini A, Diaz-Rubio E, Krell J, Dawson SJ, Raimondi C, Rutten A, Janni W, Munzone E, Carañana V, Agelaki S, Almici C, Dirix L, Solomayer EF, Zorzino L, Johannes H, Reis-Filho JS, Pantel K, Pierga JY and Michiels S: Clinical validity of circulating tumour cells in patients with metastatic breast cancer: a pooled analysis of individual patient data. Lancet Oncol 15(4): 406-414, 2014. PMID: 24636208. DOI: $10.1016 / \mathrm{S} 1470-2045(14) 70069-5$

19 Martin OA, Anderson RL, Russell PA, Cox RA, Ivashkevich A, Swierczak A, Doherty JP, Jacobs DH, Smith J, Siva S, Daly PE, Ball DL, Martin RF and MacManus MP: Mobilization of viable tumor cells into the circulation during radiation therapy. Int J Radiat Oncol Biol Phys 8(2): 95-403, 2014. PMID: 24315565. DOI: $10.1016 /$ j.ijrobp.2013.10.033

20 Martin OA, Anderson RL, Narayan K and MacManus MP: Does the mobilization of circulating tumour cells during cancer therapy cause metastasis? Nat Rev Clin Oncol 14(1): 2-44, 2017. PMID: 27550857 DOI: 10.1038/nrclinonc.2016.128

21 Yusa A, Toneri M, Masuda T, Ito S, Yamamoto S, Okochi M, Kondo N, Iwata H, Yatabe Y, Ichinosawa Y, Kinuta S, Kondo E, Honda H, Arai $\mathrm{F}$ and Nakanishi $\mathrm{H}$ : Development of a new rapid isolation device for circulating tumor cells (CTCs) using 3D palladium filter and its application for genetic analysis. PLoS One 9(2): e88821, 2014. PMID: 24523941. DOI: 10.1371/ journal.pone.0088821

22 Dejima H, Nakanishi H, Kuroda H, Yoshimura M, Sakakura N, Ueda N, Ohta Y, Tanaka R, Mori S, Yoshida T, Hida T, Sawabata N, Yatabe Y and Sakao Y: Detection of abundant megakaryocytes in pulmonary artery blood in lung cancer patients using a microfluidic platform. Lung Cancer 125: 128-135, 2018. PMID: 30429010. DOI: 10.1016/j.lungcan.2018. 09.011

23 Tsutsuyama M, Nakanishi H, Yoshimura M, Oshiro T, Kinoshita T, Komori K, Shimizu Y, Ichinosawa Y, Kinuta S, Wajima K, Sakakibara Y, Yatabe Y, Ito S and Kodera Y: Detection of circulating tumor cells in drainage venous blood from colorectal cancer patients using a new filtration and cytology-based automated platform. PLoS One 14(2): e0212221, 2019. PMID: 30811434. DOI: 10.1371/journal.pone.0212221

24 Oshima Y, Tanaka H, Murakami H, Ito Y, Furuya T, Kondo E, Kodera $\mathrm{Y}$ and Nakanishi H: Lapatinib sensitivity of two novel trastuzumab-resistant HER2 gene-amplified gastric cancer cell lines. Gastric cancer 17(3): 450-462, 2014. PMID: 23948998. DOI: $10.1007 / \mathrm{s} 10120-013-0290-6$

25 Rossi E, Basso U, Celadin R, Zilio F, Pucciarelli S, Aieta M, Barile C, Sava T, Bonciarelli G, Tumolo S, Ghiotto C, Magro C, Jirillo A, Indraccolo S, Amadori A and Zamarchi R: M30 neoepitope expression in epithelial cancer: quantification of apoptosis in circulating tumor cells by CellSearch analysis. Clin Cancer Res 16(21): 5233-5243, 2010. PMID: 20978147. DOI: 10.1158/1078-0432.CCR-10-1449

26 Yatabe Y, Hida T, Horio Y, Kosaka T, Takahashi T and Mitsudomi $\mathrm{T}$ : A rapid, sensitive assay to detect EGFR mutation in small biopsy specimens from lung cancer. J Mol Diagnostics 8(3): 335341, 2006. PMID: 16825506. DOI: 10.2353/ jmoldx.2006.050104

27 Workman P, Aboagye EO, Balkwill F, Balmain A, Bruder G, Chaplin DJ, Double JA, Everitt J, Farningham DA, Glennie MJ, Kelland LR, Robinson V, Stratford IJ, Tozer GM, Watson S, Wedge SR, Eccles SA and Committee of the National Cancer Research Institute: Guidelines for the welfare and use of animals in cancer research. Br J Cancer 102(11): 1555-1577, 2010. PMID: 20502460. DOI: 10.1038/sj.bjc.6605642

28 Hoff J: Methods of blood collection in the mouse. Lab Anim 29(10): 47-53, 2000.

29 Morton D, Abbot D, Barclay R, Ewbank R, Gask D, Heath M, Mattic S, Poole T, Seamer J, Southee J, Thompson A, Trussell B, West $\mathrm{C}$ and Jennings $\mathrm{M}$ : Removal of blood from laboratory mammals and birds. First report of the BVA/FRAME/RSPCA/ UFAW Joint Working Group on Refinement. Lab Anim 27(1): 122, 1993. PMID: 8437430. DOI: 10.1258/0023677937 81082412

30 Cassady JM, Chan KK, Floss HG and Leistner E: Recent development in the maytansinoid antitumor agents. Chem Pharm Bull (Tokyo) 52(1): 1-26, 2004. PMID: 14709862.

31 Maseki S, Ijichi K, Tanaka H, Fujii M, Hasegawa Y, Ogawa T, Murakami S, Kondo E and Nakanishi H: Acquisition of EMT phenotype in the gefitinib-resistant cells of a head and neck squamous cell carcinoma cell line through Akt/GSK-3b/snail signalling pathway. Br J Cancer 106(6): 1196-1204, 2012. PMID: 22315058. DOI: 10.1038/bjc.2012.24

32 Polioudaki H, Agelaki S, Chiotaki R, Politaki E, Mavroudis D, Matikas A, Georgoulias V and Theodoropoulos PA: Variable expression levels of keratin and vimentin reveal differential EMT status of circulating tumor cells and correlation with clinical characteristics and outcome of patients with metastatic breast cancer. BMC Cancer 15: 399, 2015. PMID: 25962645. DOI: $10.1186 / \mathrm{s} 12885-015-1386-7$

33 Vona G, Sabile A, Louha M, Sitruk V, Romana S, Schütze K, Capron F, Franco D, Pazzagli M, Vekemans M, Lacour B, Bréchot $\mathrm{C}$ and Paterlini-Bréchot $\mathrm{P}$ : Isolation by the size of epithelial tumor cells: a new method for the immunomorphological and molecular characterization of circulating tumor cells. Am J Pathol 156: 57-63, 2000. PMID: 10623654. DOI: $10.1016 / \mathrm{S} 0002-9440(10) 64706-2$

34 Desitter I, Guerrouahen BS, Benali-Furet N, Wechsler J, Ja“nne PA, Kuang Y, Yanagita M, Wang L, Berkowitz JA, Distel RJ and Cayre YE: A new device for rapid isolation by size and characterization of rare circulating tumor cells. Anticancer Res 31(2): 427-441, 2011. PMID: 21378321.

Received June 30, 2019

Revised July 19, 2019

Accepted July 22, 2019 